\title{
Simulation of Blast Waves with Headwind
}

\author{
Michael E. Olsen * \\ Scott W. Lawrence ${ }^{\dagger}$ \\ Goetz H. Klopfer $\ddagger$ \\ Donvan Mathias $\$$ \\ Jeff T. Onufer I \\ NASA Ames Research Center \\ Moffett Field, CA 94035
}

The blast wave resulting from an explosion was simulated to provide guidance for models estimating risks for human spacecraft flight. Simulations included effects of headwind on blast propogation, Blasts were modelled as an initial value problem with a uniform high energy sphere expanding into an ambient field. Both still air and cases with headwind were calculated.

\section{Nomenclature}

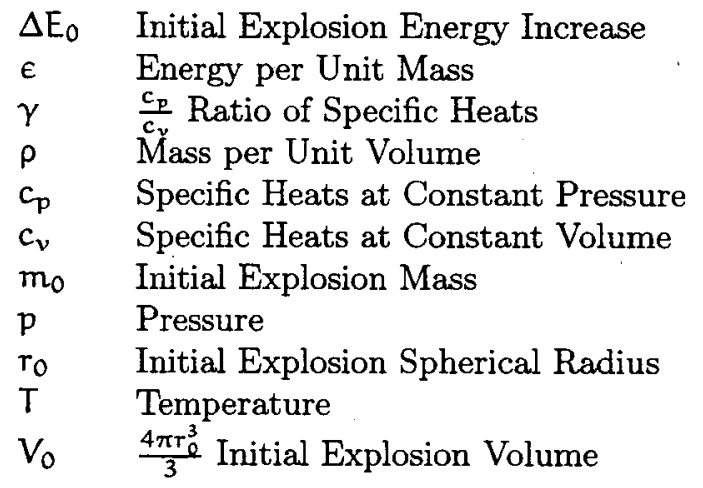

\section{Introduction}

Requirements for a abort system for a launch vehicle are driven in part by the necessity to escape a blast wave resulting from a system malfunction. Blast wave propogation is affected by the vehicle speed, altitude, the extent of material involved in the blast and its spatial distribution. Prediction of blast wave overpressures and arrival times are important in deriving requirements for abort system thrust, trajectory and abort lead times. An investigation of blast wave propogation is ongoing as part of a probablistic risk assessment (PRA) project.

\footnotetext{
* Research Scientist, NASA Ames Research Center, Associate Fellow AIAA

${ }^{\dagger}$ Research Scientist, NASA Ames Research Center

$\ddagger$ Research Scientist, NASA Ames Research Center

$\$$ Research Scientist, NASA Ames Research Center, Senior Member AIAA

TResearch Scientist, NASA Ames Research Center
} 


\section{Method}

\section{A. Euler Equations}

The Euler equations, written in conservation law form are

$$
\frac{\partial Q}{\partial t}+\frac{\partial F_{i}}{\partial x_{i}}=0
$$

Where

$$
\begin{aligned}
& Q=\left[\rho, \rho u_{1}, \rho u_{2}, \rho u_{3}, \rho \varepsilon_{T},\right] \\
& F_{1}=\left[\rho u_{1}, \rho u_{1}^{2}+p, \rho u_{1} u_{2}, \rho u_{1} u_{3}, \rho u_{1}\left(\varepsilon_{T}+p\right)\right] \\
& F_{2}=\left[\rho u_{2}, \rho u_{2} u_{1}, \rho u_{2}^{2}+p, \rho u_{2} u_{3}, \rho u_{2}\left(\varepsilon_{T}+p\right)\right] \\
& F_{3}=\left[\rho u_{3}, \rho u_{3} u_{1}, \rho u_{3} u_{2}, \rho u_{3}^{2}+\tilde{p}, \rho u_{3}(\varepsilon T+p)\right]
\end{aligned}
$$

$$
\text { where: } \quad \varepsilon_{t}=\varepsilon+\left(u_{i} u_{i}\right) / 2 \quad \varepsilon=\int_{0}^{T} c_{\nu} d T=c_{v} T \quad p=(\gamma-1) \rho \varepsilon
$$

\section{B. Numerical Method}

The code used in this study was OVERFLOW2. Matrix dissipation was used with smoothing parameters as recomended by earlier studies of high speed flows with this code. ${ }^{1}$ The recommended eigenvalue limits $\left(V_{\epsilon_{n}}=0.3, V_{\epsilon_{1}}=0.3\right)$ are not adjusted.

Time accurate simulations were obtained with the dual time method. In general, simulations were run at the highest physical time step possible to allow the initial condition to be simulated stably. This is a conservative choice, as the simulation can be run at much higher time steps once the high variation initial transients integrated past.

The relaxation method is the implicit Pulliam Chausee diagonal method, with timesteps chosen for a constant CFL (itime=3). CFL values for these high speed flows were chosen at 0.4 , and the variable time step was adjusted as described in the overflow documentation. ${ }^{2}$

\section{Scales, Nondimensional and Dimensional Coordinates}

A length scale of the problem is given by the cube root of the ratio of the explosion energy to the ambient pressure. Thus, $\lambda=\left(E_{0} / p_{a}\right)^{1 / 3}$ is a length which is a natural length scale of the problem. A natural velocity scale is given by the ratio of the initial energy to the initial mass. For example, a TNT explosion has a natural velocity scale of $\left.2.044 \mathrm{~km} / \mathrm{sec}\left(4.18 \times 10^{6}\right)^{1 / 2}\right)$. Pressures are non-dimensionalized by the ambient pressure.

To translate a solution from the non-dimensional overflow coordinates, the length scale of the blast in physical coordinates is determined by the initial radius in computational coordinates and the corresponding physical extent of the initial sphere. Given the ratio of the explosion to ambient density, and the initial mass, the physical size of the sphere in meters is given by

$$
r_{0}=\left(\frac{\rho_{a}}{\rho_{0}} \frac{3 m_{0}}{4 \pi r h o_{a}}\right)^{1 / 3}
$$

Thus, if the initial radius of the sphere is taken as unity in the non-dimensional computational coordinates, the physical length $\mathrm{L}$ corresponding with this length is given by this formula. The time scales (which in overflow are given by $L / a_{\infty}$ ) are then determined by this choice of $L$ and the ambient sound speed. The reference Mach number is taken as unity, so that $u_{\infty}=a_{\infty}$. Pressures, temperatures and velocities follow the standard overflow methodology, where the 'infinity' conditions are identified with ambient conditions. 


\section{Iniitial Condition}

The initial condition was simulated as a sphere of high energy fluid at rest with respect to the inertial frame chosen for the simulation. Three parameters fully specify the conditions within the initial sphere containing the explosion, the additional energy $\Delta E_{0}$, the contained mass, $m_{0}$, and the initial radius, $r_{0}$. Assuming a perfect gas, the total energy in a sphere is given by

$$
\begin{aligned}
E_{0} & =\frac{4 \pi r_{0}^{3}}{3} \rho_{0} c_{\nu} T_{0} \\
& =m_{0} c_{\nu} T_{0}
\end{aligned}
$$

The energy $\left(\Delta E_{0}\right)$ and mass $\left(m_{0}\right)$ are taken as known inputs to the simulation, and it is assumed that before the explosion the mass involved was already at ambient temperature, so that $E_{0}=\rho_{0} c_{v} T_{a}+\Delta E_{0}$, and thus the energy per unit mass $\left(\frac{E_{0}}{m_{0}}=\epsilon\right)$ is a constant for a given explosive yield and mass, If the perfect gas assumption is made, this implies that the initial temperature of the ball is similarly a function of these two parameters. Given a more realistic assumption of chemically reacting gases, assuming chemical equilibrium and given element mass fractions, the temperature is similarly a function of these two parameters.

The initial density of the ball is given by $\rho_{0}=4 \pi \mathrm{m}_{0} \mathrm{r}_{0}^{3} / 3$. given a fixed mass, then the density is a function of $r_{0}$, the initial extent of the explosion. The range of values plausible for density range from water on the high end(as kerosene is close to a specific gravity of one), down to the ambient atmospheric density. The high range corresponds to a condition where the fuel and oxidizer are perfectly mixed, and then ignited simultaneously. This is admittedly an unrealistic possibility, but is a plausible upper limit on the density likely to be encountered in any explosion. The lower limit would correspond to the fuel mixing and expanding before ignition to the ambient pressure (and having a molecular weight comparable to that of air), and then igniting simultaneously. Again, this is not a plausible scenario, but again provides a plausible lower bound. The sensitivity of the solutions to this range of initial densities is explored, and is found to be a relatively small source of variation in blast overpressure predictions.

These blast calculations are intended to provide apply to conditions where a given mass of the fuel available is ignited, but not all the mass. The spatial extent of the fuel which is ignited then determines the amount of mass which should be used as $m_{0}$ which could range from a minimum of the amount necessary to provide the $\Delta E_{0}$ (which assumes that all the fuel in a given volume is burnt along with the required amount of oxygen), to an essentially unbounded amount (allowing for the mixing of the fuel with the ambient air before ignition). The standard assumption used is that the total mass involved is equal to the mass of propellant available.

Varying $m_{0}$ holding $\Delta \mathrm{E}_{0}$ fixed varies the temperature(which is given by $\Delta \mathrm{T}_{0}=\Delta \mathrm{E}_{0} /\left(\mathrm{c}_{v} \mathrm{~m}_{0}\right)$ ). Holding the density fxed (so that $r_{0}$ varies) will then correspond to assuming that the density of the initial fuel/oxygen combination is roughly at fixed density before ignition, and varying $m_{0}$ is simply including more or less unburnt material in the initial explosion sphere. This type of parameter variation will be termed a 'constant density' variation.

Holding $m_{0}$ and $\Delta E_{0}$ fixed, but varying $r_{0}$ corresponds to choosing varying initial densities, and results in a sphere with at fixed temperature (given by Delta $T_{0}=\Delta E_{0} /\left(c_{v} m_{0}\right)$ ) but with variation in the initial density $\rho_{0}=3 \mathrm{~m}_{0} /\left(4 \pi r_{0}^{3}\right)$. This parameter variation will be termed a 'constant temperature' variation.

Holding $r_{0}$ and $\Delta E_{0}$ fixed, but varying $m_{0}$ results in a sphere at a fixed pressure(given by $p=3(\gamma-$ 1) $\left.\mathrm{E}_{0} /\left(4 \pi \mathrm{r}_{0}^{3}\right)\right)$ but with varying temperature and pressure. This parameter variation will be termed a 'constant pressure' variation.

\section{Results}

\section{A. Comparison with Experiment}

One experiment which can be used for validation purposes for this case is the Boyer experiment. ${ }^{3}$ This is an experiment with a glass sphere filled with either air or helium is shattered, This is a particularly valuable set of data because it has a well defined set of intial conditions. The pressure ratio and temperature ratio(which is unity) are known, and the initial condition is still air within the high energy sphere is zero velocity. A wealth of data is available for this case, including what are effectively experimentally determined $x-$ t diagrams which allow the blast wave speed predictions to be verified along with the overpressures as a function of distance. 
The overpresssure for the case with air $(\gamma=1.4)$ at a pressure ratio of 27.2(or 22 corrected) is compared with the results of the simulation in figure 1. The solid line is the line corresponding to the maximum pressure over the span of the simulation at the given spatial location. This was obtained by taking obtaining what would be equivalent to oscilliscope traces, the solution as a function of time at a given spatial location, then searching this trace for the maximum pressure. The experimental pressures are given by symbols, and snapsots of the pressure field predictions at given times are superimposed, showing the predictions for the blast wave propogation. The experimental results were corrected to account for the energy loss created by accelerating the shattered remains of the glass sphere. The experimental pressure measurements lie within the range of pressures bounded by the uncorrected and corrected pressure ratio results.

The blast wave arrival time are another important prediction which the simulation will provide. The experimental time have

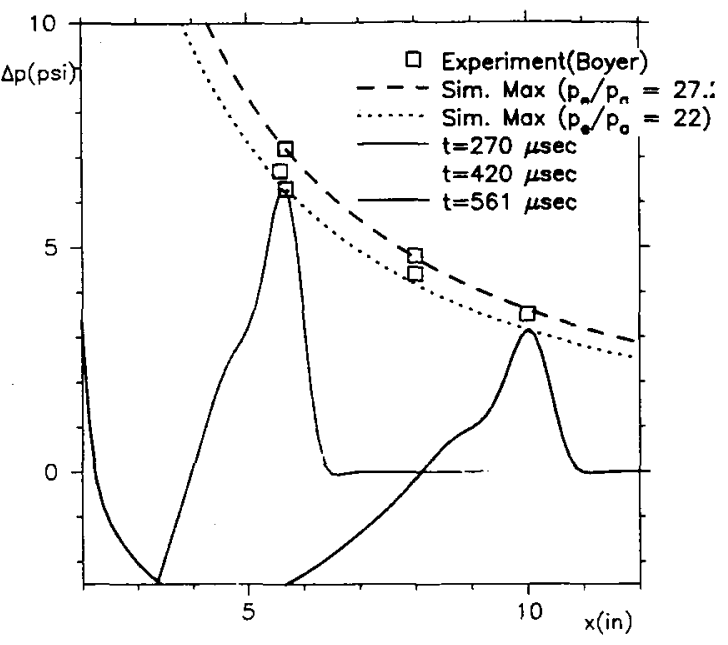

Figure 1. Overpressure Predictions for Boyer Air Blast a delay of 50 to $60 \mu$ seconds between the mallet striking the glass sphere and the emergence of the shock wave from in the schlieren record. It is assumed that this is the time required for the glass sphere to absorb the energy of the mallet and shatter. The arrival time for the blast wave at 5.7 inches is between $300 \mu \mathrm{sec}$ and $320 \mu \mathrm{sec}$, which are predicted to be at 240 to $270 \mu \mathrm{sec}$ computationally. Given the delay time of 50-60 $\mu$ seconds for the glass enclosure to shatter, this is good agreement with the experimental measurements. The arrival time at 8 inches is $440 \mu s e c$ experimentally, and this simulation prediction is between and 380 and $420 \mu \mathrm{sec}$, again in good agreement when the glass shattering delay is taken into account. A possibly unwarranted extrapolation from the experimental $x-t$ diagram has an arrival time at 10 inches of about $590 \mu$ seconds, which again is in good agreement, with the predicted 510 to $540 \mu$ seconds arrival time.

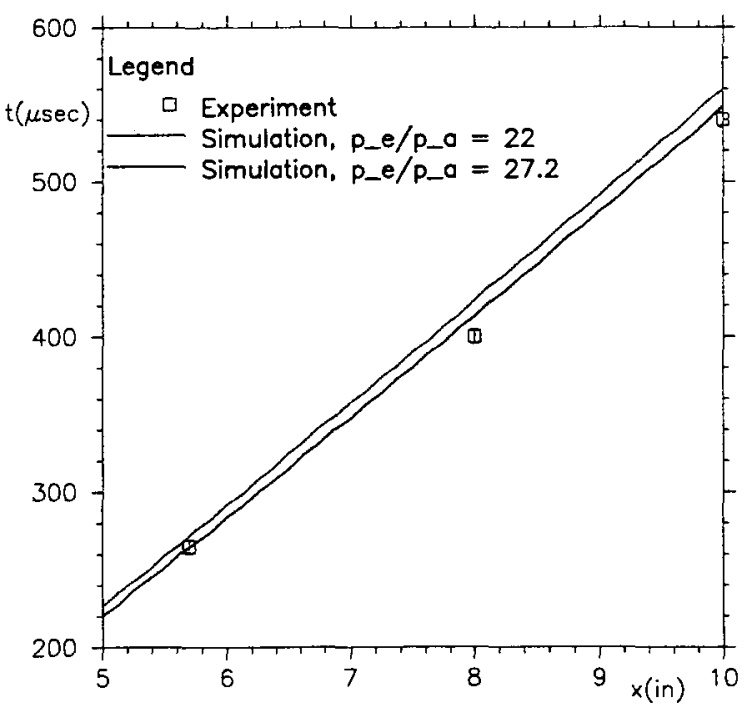

(a) Primary Blast Wave Arrival

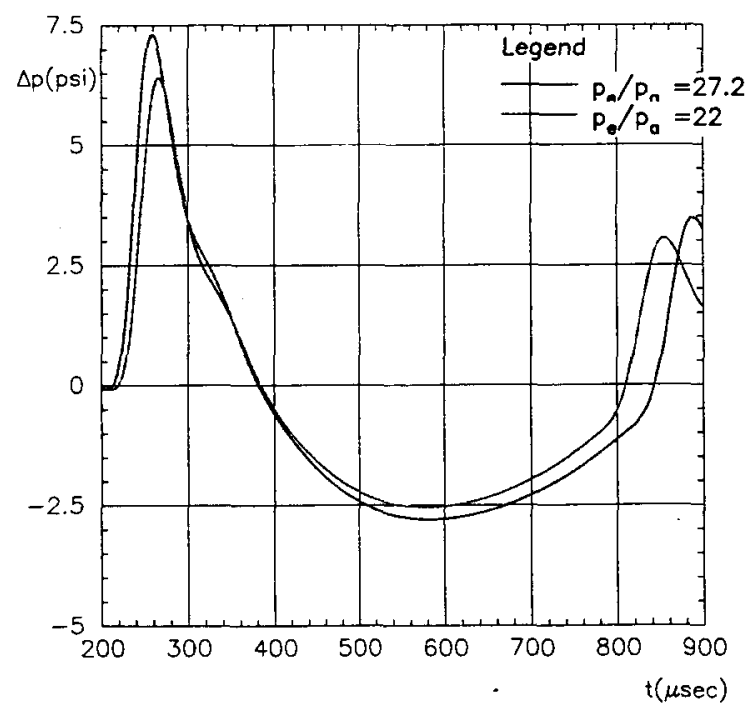

(b) Primary/Secondary Blast Wave Arrivals

Figure 2. Comparison of Arrival Time Predictions with Boyer Experiment

Another 'arrival time' prediction is for the time delay between the arrival of the primary and secondary shock at the $\mathrm{r}=5$.7in postion. The time delay between the arrival of these two shocks may be obtained the the experimental oscilliscope trace(Figure 2(a)), and is $580 \mu \mathrm{sec}$ experimentally. The computational oscilliscope trace is shown in figure 2(b), and the delay between $580 \mu \mathrm{sec}$ and $610 \mu \mathrm{sec}$. 


\section{B. Explosions in Still Air}

The initial solutions obtained were obtained for still air conditions. For an explosion with an initial energy of $1 \mathrm{KT}$ of TNT (equivalent to 3.8 terajoules), with a mass of $5 \mathrm{KT}$ (note that the mass is assumed to be five times the mass of the TNT equivalent), this yields an internal energy of $836 \mathrm{KJ}$ oules $/ \mathrm{kg}$. Assuming a molecular wieght of $29 \mathrm{~kg} / \mathrm{kMole}$, a ratio of specific heats of 1.4, this yields a temperature increase of

$$
\begin{aligned}
\Delta T & =\frac{\Delta E_{0}}{m_{0}} \frac{(\gamma-1) \mathcal{M}}{\mathcal{R}} \\
& =836 \times 10^{3} \frac{29(0.4)}{8314.3}{ }^{\circ} \mathrm{C} \\
& =1164.3^{\circ} \mathrm{C}
\end{aligned}
$$

again assuming perfect gas conditions. Note that lowering $\gamma$ or increasing the mass decreases this temperature increase. For instance, if the same calculation were done for TNT, where the mass was $1 \mathrm{KT}$, but $\gamma$ was decreased to 1.3(to account for non-perfect gas behaviour), the corresponding temperature increase is $4,545^{\circ} \mathrm{C}$, substantially hotter.

The overpressure for the $1 \mathrm{KT}$ TNT energy with $5 \mathrm{KT}$ mass case in universal coordinates is given in figure 3 (c) for a variety of density assumptions. The resulting variation in overpressure in confined to the nearfield, and is much smaller than the corresponding overpressure variation shown by a variation in either explosive yield or mass in figure $3(\mathrm{~b})$.

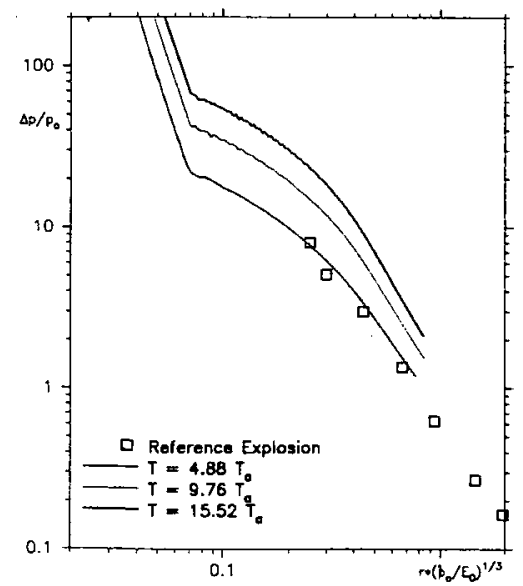

(a) Constant
Pressure( $E_{0}, r_{0}$ Constant $)$

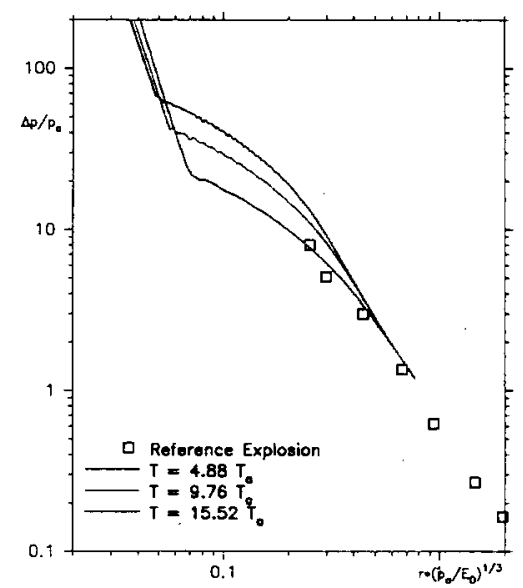

$\begin{array}{ll}\text { (b) Constant } & \text { Initial } \\ \text { Density }\left(m_{0}, r_{0} \text { Constant }\right) & \end{array}$

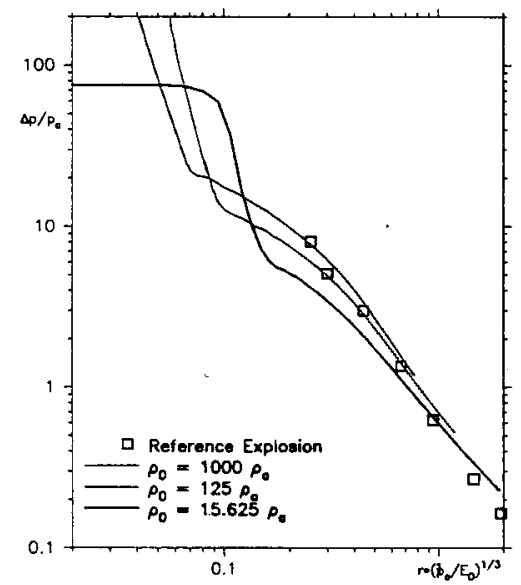

(c) Constant Initial Temperature( $E_{0}, m_{0}$ Constant)

Figure 3. Overpressure Predictions for nominal 1KT TNT Explosion, with 5KT Mass

\section{Explosions in Headwinds}

The impetus for this study was to provide an check on estimates of the effects of headwind on blast pressures. Representative plots of overpressure predicitions for blasts occuring in the presence of a headwind are shown in figure 4. Figure 4(a) shows the effect of lowering the mass of the initial blast, keeping the explosive energy constant. This yields higher overpressures initially, but the shock wave actually does not extend as far upstream as the case with the higher mass. Figure 4(b) shows the more subtle effect of varying the spatial extent of the initial blast, holding both blast energy and initial mass constant. There are some initial differences in overpressure, but they are much less pronounced and both cases 'turn around' at the same location.

One of the features of the case with headwind not present in the still air case is the finite extent of influence in the upwind direction of the blast. This critical difference is one of the reasons for doing this study, which is to better elucidate the escape system requirements for potential launch vehicles. 


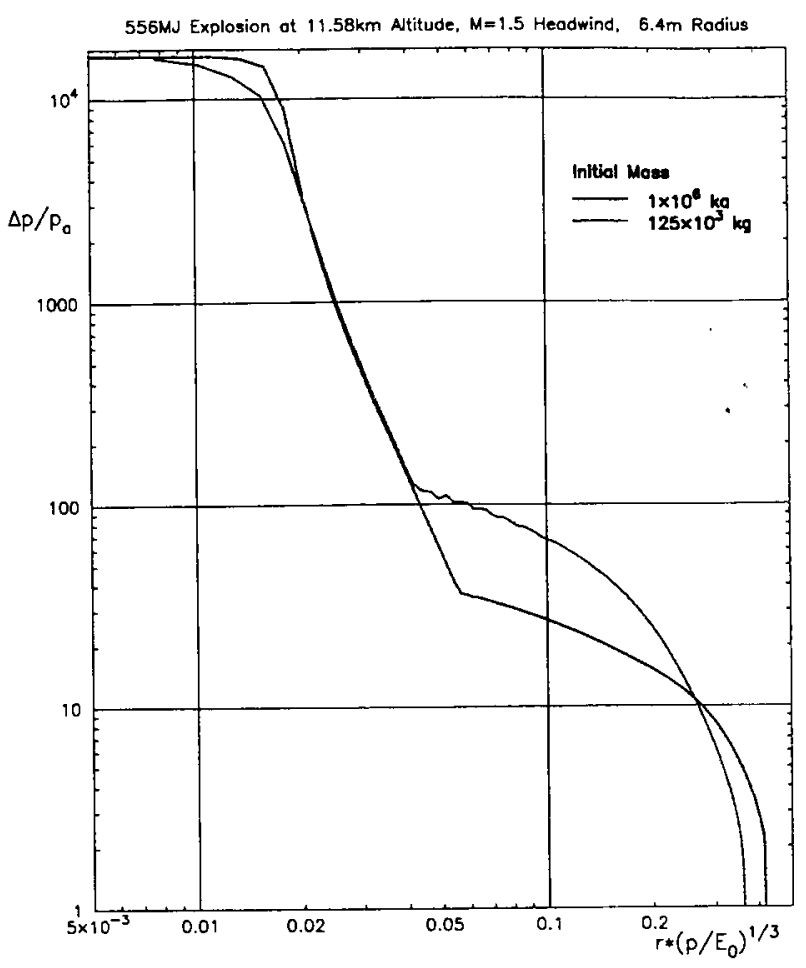

(a) Varying Initial Mass

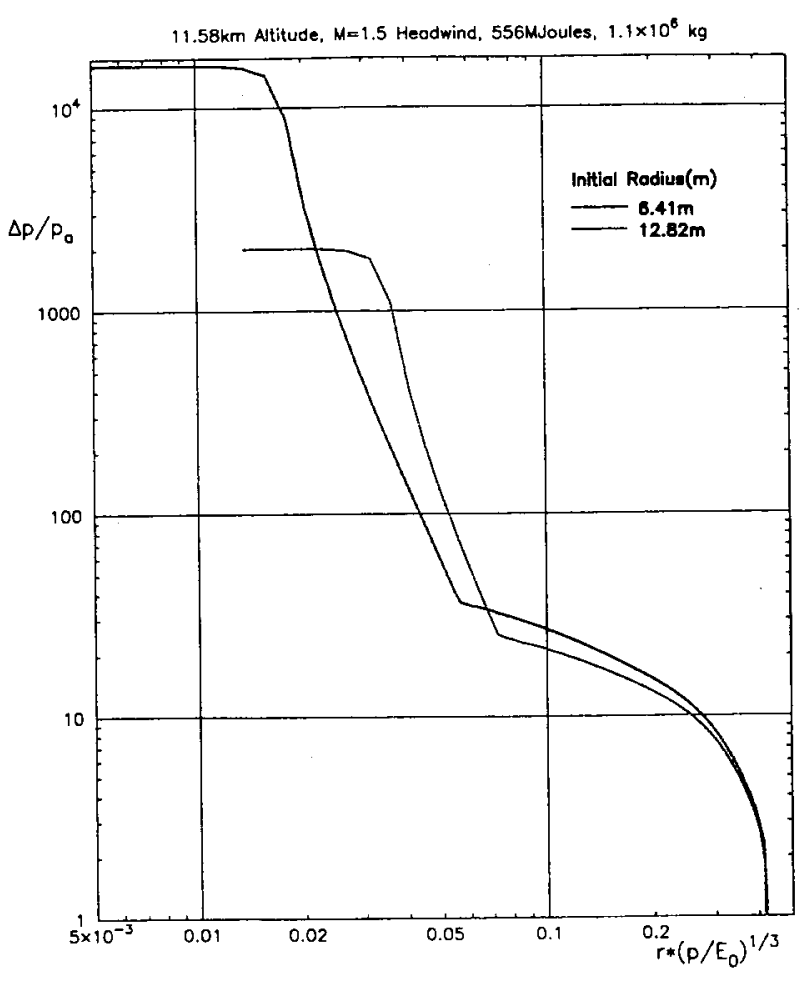

(b) Varying Initial Density

Figure 4. Overpressure Predictions for Nominal 141 Ton TNT Explosion with Headwind

\section{Plans}

Reacting flow simulations are planned to elucidate some rudimentary effects of finite rate chemistry and caloric imperfection on the blast wave propogation. Conditions at various altitudes, various headwinds, and various explosive yield/mass combinations are being investigated, and will be reported. Overpressures produced on vehicles will be investigated to further elucidate the blast wave effects on crew safety.

\section{References}

'Olsen, Michael E. and Dinesh K. Prabhu. "Application of OVERFLOW to Hypersonic Perfect Gas Flowfields". AIAA Paper 2001-2664, 2001

${ }^{2}$ Buning, Pieter G. et al. Overflow user's manual. Version 1.8, NASA Ames Research Center, February 1998.

${ }^{3} \mathrm{D}$. W. Boyer. "An experimental study of the explosion generated by a pressurized sphere ". Journal of Fluid Mechanics, 9:401-429, 1960. 